\title{
ANÁLISE DA MATURIDADE E DO PERFIL DE PROGRAMAS DE GESTÃO DO CONHECIMENTO: PESQUISA EXPLORATÓRIA E COMPARATIVA EM ORGANIZAÇÕES BRASILEIRAS E PORTUGUESAS
}

\section{A MATURITY ANALYSIS OF KNOWLEDGE MANAGEMENT PROGRAMS: EXPLORATORY AND COMPARATIVE SURVEY OF BRAZILIAN AND PORTUGUESE ORGANIZATIONS}

\author{
Rodrigo Baroni de Carvalho'; Marta Araújo Tavares Ferreira ${ }^{2}$; Ricardo Vidigal da Silva ${ }^{3}$ \\ ${ }^{1}$ Universidade FUMEC - Belo Horizonte - Brasil baroni@,fumec.com.br \\ ${ }^{2}$ Universidade Federal de Minas Gerais / Centro Universitário U.N.A - Belo Horizonte - Brasil \\ maraujo@eci.ufmg.br \\ ${ }^{3}$ Universidade de Évora - Évora - Portugal ricardo.silva@mail.telepac.pt
}

\begin{abstract}
Resumo
As organizações têm implantado iniciativas de Gestão do Conhecimento cujo leque de objetivos engloba a preservação da memória organizacional, a melhoria da produtividade dos trabalhadores do conhecimento, o estímulo ao processo de inovação e o aumento da competitividade. Entretanto, a avaliação dos resultados obtidos nesses programas é uma tarefa árdua devido à falta de padrões para implementação das abordagens teóricas e à natureza complexa e interdisciplinar da Gestão do Conhecimento. Tanto o meio empresarial quanto a comunidade acadêmica têm se mobilizado a debater essas questões e propor modelos que auxiliem as organizações no desenvolvimento de seus programas de Gestão do Conhecimento. O artigo sugere uma combinação do modelo acadêmico da organização do conhecimento com o modelo comercial KMMM (Knowledge Management Maturity Model). $O$ modelo resultante foi utilizado em uma survey realizada com 98 organizações brasileiras e 70 portuguesas. As variáveis do modelo estão relacionadas a aspectos como cultura colaborativa, inteligência competitiva, tomada de decisão, criação do conhecimento, estratégia e comunidades de prática. O modelo proposto pretende auxiliar as organizações na identificação de pontos fortes e fracos das suas práticas relacionadas com a gestão do conhecimento.
\end{abstract}

Palavras-chave: gestão do conhecimento, inovação, estratégia, tomada de decisão.

\section{Introdução}

$\mathrm{Na}$ literatura especializada, são encontradas diversas propostas de modelos para a gestão do conhecimento organizacional. Após analisar inúmeras propostas de técnicas e práticas gerenciais modernas, RODRIGUES et al. (2003) constataram que o maior número de propostas está relacionado à inovação e ao conhecimento. Essa diversidade de propostas é mais marcada pela complementaridade do que pelo antagonismo. Um aspecto comum entre os modelos existentes é o 
reconhecimento do papel estratégico do conhecimento na competitividade das empresas. No entanto, não basta apenas saber que o conhecimento é um recurso importante para a organização. É preciso mudar de atitude após a constatação desse fato. Assim sendo, a literatura especializada propõe práticas sistemáticas e ações planejadas para facilitar, gerenciar e alavancar o conhecimento organizacional.

No entanto, várias dúvidas surgem quando as organizações buscam implementar práticas de gestão do conhecimento. O artigo sugere uma síntese de um modelo de maturidade (EHMS e LANGEN, 2002) com o modelo de Organização do Conhecimento (CHOO, 1998) e aplica este modelo consolidado para avaliar a existência de práticas de gestão do conhecimento em organizações brasileiras e portuguesas.

$\mathrm{O}$ artigo está organizado da seguinte forma: o capítulo 2 do artigo apresenta os modelos de gestão do conhecimento (GC) que constituem os pilares da revisão teórica. O capítulo 3 descreve a metodologia empregada. No capítulo 4, é feita a discussão dos resultados obtidos em uma survey em 98 organizações brasileiras e 70 portuguesas. A conclusão sintetiza os principais pontos e sugere trabalhos futuros.

\section{Modelos de Maturidade em Gestão do Conhecimento}

As diferenças entre os modelos teóricos de gestão do conhecimento residem na ênfase que é dada a certos aspectos do conhecimento organizacional. Alguns modelos priorizam a geração do novo conhecimento, a criatividade, a inovação, enquanto que outros modelos destacam a preservação e a codificação do conhecimento já existente. Certos modelos enfatizam o conhecimento interno à empresa e a percepção do conhecimento como estoque. Já outros autores descrevem o conhecimento como fluxo e desenvolvem modelos que facilitam a troca de conhecimento em toda a cadeia de valor da organização. Os modelos associados ao capital intelectual são influenciados por conceitos contábeis e se preocupam mais com a definição de indicadores gerenciais e a mensuração dos ativos intangíveis. Os modelos associados à gestão do conhecimento por sua vez se concentram nos processos vinculados ao conhecimento organizacional tais como criação, conversão, codificação entre outros.

De acordo com Teixeira (2000, p.11), a gestão do conhecimento é "uma certa forma de olhar a organização em busca de pontos dos processos de negócios em que o conhecimento possa ser usado como diferencial, envolvendo o conhecimento útil, oriundo da experiência, da análise, da pesquisa, do estudo, da inovação, da criatividade, conhecimento sobre mercado, concorrência, clientes, processos e tecnologia". 
Adotando uma abordagem bastante pragmática, Davenport e Prusak (1998, p.61) conceituam a gestão do conhecimento como sendo o conjunto de atividades relacionadas à geração, codificação e transferência do conhecimento. Os autores reconhecem que a temática do conhecimento não constitui novidade, mas a proposta de uma forma de gerenciá-lo é inovadora e útil, pois muitas empresas perceberam que necessitam de mais do que apenas uma abordagem aleatória (e até mesmo inconsciente) do conhecimento corporativo para vencer na economia atual e futura.

Entre os diversos modelos teóricos existentes, tais como Leonard-Barton (1995), Nonaka e Takeuchi (1995), Davenport e Prusak (1998), Terra (2000) e Krogh et al. (2001), optou-se por utilizar o modelo da organização do conhecimento (knowing organization) proposto por Choo (1998), devido ao fato de estar teoricamente muito bem fundamentado na relação existente entre a gestão da informação e a gestão do conhecimento. Além disso, esse modelo foi recentemente aplicado por Burrows (2005) para avaliar práticas de GC na China.

O modelo propõe o ciclo do conhecimento que aborda o uso da informação nos processos de construção de sentido (sense making), criação de conhecimento e tomada de decisão. O modelo é explicitamente definido da seguinte maneira:

No modelo da organização do conhecimento, a construção de sentido, a criação de conhecimento e a tomada de decisões são atividades socialmente distribuídas por muitos níveis e funções da organização. O conhecimento organizacional é a propriedade que emerge da rede de processos de uso da informação por meio da qual a organização constrói significados comuns sobre sua identidade e sua atividade; descobre, partilha e aplica novos conhecimentos; e inicia ações padronizadas por meio da busca, da avaliação e da seleção de alternativas CHOO (2003, p.370).

A construção de sentido está vinculada com a maneira que a organização interpreta o ambiente e produz significados compartilhados internamente sobre o mesmo. Já a criação de conhecimento ocorre através da conversão e compartilhamento de diferentes formas do conhecimento organizacional, seja tácito ou explícito, resultando em novas capacidades e inovação. Por fim, a organização analisa informações e toma decisões através do uso de regras e rotinas que buscam reduzir a complexidade e a incerteza. No âmbito da GC, a tomada de decisão está relacionada com a aplicação prática e uso efetivo do conhecimento.

Os modelos de maturidade de GC recebem inspirações principalmente dos modelos de controle de qualidade e do modelo CMM (Capability Maturity Model) de maturidade do desenvolvimento de software, que foi proposto pelo SEI (Software Engineering Institute) da universidade de Carnegie Mellon (PAULK et al., 1995). Entretanto, quando comparado a um assunto como engenharia de software, percebe-se que o domínio da GC compreende um leque mais amplo de aspectos sócio-culturais. 
Por outro lado, Weber et al. (2002) defendem a postura de que a existência de padrões para GC permitirá que trabalhos futuros comecem em um patamar mais alto, evitando a reinvenção da roda que a própria GC tanto critica. De acordo com os autores, o primeiro passo rumo a uma GC menos amadora e mais profissional consiste na avaliação sistemática das iniciativas organizacionais de GC, permitindo a identificação de problemas e a realização de ajustes.

Entre os modelos mais conhecidos e utilizados pelos praticantes de GC, podem ser destacados a metodologia para resultados em GC (roadmap to KM results) do APQC (American Productivity \& Quality Center) e o modelo de maturidade de GC (Knowledge Management Maturity Model) desenvolvido pela Siemens. Apesar do modelo KMMM ter sido um proposto por uma empresa e não por um organismo independente, optou-se por adotá-lo nessa pesquisa pelo fato de ser o mais abrangente e mais detalhado. Para permitir um melhor equilíbrio entre teoria e prática, optou-se por utilizar uma síntese de um modelo acadêmico (CHOO, 1998) com um modelo de mercado proposto pela Siemens (EHMS e LANGEN, 2002).

Em um modelo de maturidade, os níveis são caracterizados por requisitos específicos que devem ser atingidos em cada nível e é altamente improvável conseguir pular um nível em um processo evolutivo. De acordo com Ehms e Langen (2002), os níveis do KMMM podem ser assim descritos:

- Inicial: os processos de GC não são conscientemente controlados, não existe uma linguagem para descrever problemas a partir de uma perspectiva do conhecimento e tarefas intensivas em conhecimento não são percebidas como críticas para o sucesso da organização;

- Repetitível: existência de profetas de GC, surgimento de projetos-piloto de GC, existência de dúvidas sobre o sucesso ou fracasso desses projetos e percepção crescente de práticas de GC embutidas nos processos de negócio;

- Definido: existência de práticas de GC estáveis associadas ao dia-a-dia da organização e criação de funções associadas à GC;

- Gerenciado: mensuração de indicadores de GC e existência de uma estratégia de GC aplicada em toda a organização;

- Otimizado: utilização das métricas coletadas no nível 4 para calibrar o modelo de GC.

O modelo também possui oito áreas-chave que são avaliadas em cada nível, sendo que, segundo Ehms e Langen (2002), as seguintes áreas-chave funcionam como dimensões da GC:

- Estratégia: aspectos relacionados com a definição de uma estratégia corporativa, de objetivos específicos e de um orçamento para as iniciativas de GC;

- Ambiente e parcerias: aspectos relacionados à obtenção e uso de conhecimento externo à organização (clientes, parceiros, fornecedores); 
- Pessoas e competências: inclui tópicos de RH (Recursos Humanos) como seleção de pessoal, desenvolvimento de carreira e gestão de competências;

- Colaboração e cultura: abrange aspectos como cultura organizacional, comunicação e estrutura de equipes de trabalho;

- Liderança e suporte: diz respeito ao apoio das gerências de nível médio às práticas de GC;

- Estruturas do conhecimento: tópicos associados à gestão do conteúdo e organização das bases de conhecimento organizacional;

- Tecnologia e infra-estrutura: suporte de TI (Tecnologia da Informação) para as práticas de $\mathrm{GC}$;

- Processos e papéis: definição de papéis específicos para suporte à GC.

\section{Metdologia de Pesquisa}

O objetivo da pesquisa exploratória foi realizar uma avaliação da existência de práticas de gestão do conhecimento em organizações brasileiras e portuguesas. O modelo adotado na pesquisa foi resultante da combinação do modelo da organização do conhecimento proposto por Choo (1998) com o modelo KMMM desenvolvido na Siemens por Ehms e Langen (2002). A área-chave do KMMM denominada de "Tecnologia e infra-estrutura" foi excluída desse estudo, pois o grupo de pesquisa está desenvolvendo um outro estudo paralelo, onde o uso das tecnologias de apoio à GC é investigado em maior detalhe com um questionário dirigido para o gestor de TI. Várias áreas do KMMM são semelhantes às dimensões da organização do conhecimento. A Tabela 1 apresenta a combinação entre os dois modelos, nomeando as variáveis a serem investigadas.

Tabela 1 - Variáveis relacionadas às práticas de gestão do conhecimento

\begin{tabular}{cll}
\hline \multicolumn{1}{c}{ Variável } & \multicolumn{1}{c}{$\begin{array}{c}\text { Dimensões da organização do } \\
\text { conhecimento } \\
(\mathrm{CHOO}, 1998)\end{array}$} & $\begin{array}{c}\text { Áreas-chave da maturidade em GC } \\
\text { (EHMS e LANGEN, 2002) }\end{array}$ \\
\hline Inteligência Competitiva $(\mathrm{gc} 1)$ & Construção do sentido & Ambiente e parcerias \\
\hline Orçamento de GC $(\mathrm{gc} 2)$ & Construção do sentido & Estratégia \\
\hline Estratégia de GC $(\mathrm{gc} 3)$ & Criação do conhecimento & Colaboração e cultura \\
\hline Cultura colaborativa $(\mathrm{gc4})$ & Criação do conhecimento & Colaboração e cultura \\
\hline Comunidades de práticas $(\mathrm{gc5})$ & Colaboração e cultura \\
\hline $\begin{array}{c}\text { Socialização do conhecimento } \\
(\mathrm{gc} 6)\end{array}$ & Criação do conhecimento & Estruturas do conhecimento \\
\hline Registro de lições aprendidas $(\mathrm{gc} 7)$ & Criação do conhecimento & Pessoas e competências \\
\hline $\begin{array}{c}\text { Avaliação de atitudes de } \\
\text { compartilhamento }(\mathrm{gc} 8)\end{array}$ & Criação do conhecimento & Liderança e suporte + processos e papéis \\
\hline Liderança de GC $(\mathrm{gc} 9)$ & & Liderança e suporte + processos e papéis \\
\hline Projetos piloto de GC $(\mathrm{gc} 10)$ & & Mensuração \\
\hline Mensuração $(\mathrm{gc} 11)$ & & \\
\hline Tomada de decisão $(\mathrm{gc} 12)$ & Tomada de decisão & \\
\hline
\end{tabular}


As práticas selecionadas foram traduzidas em um questionário de forma a gerar um instrumento de pesquisa. O questionário foi submetido a um processo de revisão por um grupo composto por 3 professores doutores, 1 professor com mestrado e 2 estudantes de doutorado. A seguir, para efeito de pré-teste, o questionário foi aplicado em duas organizações brasileiras: um banco público e uma indústria química. Essas duas etapas contribuíram para o refinamento do questionário, resultando na revisão dos enunciados, na inclusão e exclusão de questões.

O questionário utilizou uma escala Likert de 11 pontos, com os extremos "discordo totalmente" e "concordo totalmente". O questionário foi convertido em um conjunto de páginas Web, sendo que as respostas foram armazenadas em um banco de dados relacional. A parte inicial do questionário diz respeito às variáveis funcionais e a segunda parte contém questões de cunho sócio-geográfico. O Anexo 1 apresenta um extrato sintético do questionário. É curioso comentar que foram feitas, em endereços Web distintos, duas versões do questionário: uma em português do Brasil e outra em português de Portugal. Pretendeu-se dessa forma deixar os respondentes portugueses mais à vontade com o uso de termos mais familiares. Por exemplo, em Portugal, o termo "inquérito" é mais usual do que "questionário" para pesquisas científicas.

As organizações brasileiras participantes da pesquisa foram escolhidas através de uma amostra por conveniência composta por 353 membros de uma lista de discussão sobre intranets e portais (lista wi-intranet), 890 membros de uma lista de discussão sobre gestão do conhecimento (competitive-knowledge), 1677 membros da lista de discussão da Sociedade Brasileira de Gestão do Conhecimento, aproximadamente 400 membros da mala direta da Associação Portuguesa de Gestão do Conhecimento e por 45 contatos pessoais dos autores com gestores de portais, totalizando um universo de 3.365 pessoas. O universo real é difícil calcular porque várias pessoas fazem parte de mais de uma dessas listas de discussão e por questões de privacidade não foi obtido o e-mail dos membros de cada lista.

\section{Análise dos Resultados}

No $1^{\mathrm{o}}$. semestre de 2005, foram obtidas 168 respostas (98 do Brasil e 70 de Portugal), resultando em uma taxa de retorno estimada de 4,99\%. Como compensação, os participantes de cada lista de discussão concorreram ao sorteio de 10 livros de autoria de um dos pesquisadores. Entre as organizações respondentes, 46,4\% têm mais de mil funcionários e 85,1\% têm mais de 100 funcionários. A Tabela 2 descreve o perfil das organizações por setor da economia, em ordem decrescente do número de empresas por setor. 
Tabela - Perfil dos respondentes por setor de atividade

\begin{tabular}{lcc}
\hline Setor de Atividade & Número de Empresas & \% Respondentes \\
\hline Governo & 27 & $16.1 \%$ \\
\hline Tecnologia da Informação & 22 & $13.1 \%$ \\
\hline Setor financeiro (bancos, corretoras) & 17 & $10.1 \%$ \\
\hline Indústria química e petróleo & 13 & $7.7 \%$ \\
\hline Educação & 11 & $6.5 \%$ \\
\hline Telecomunicações & 10 & $6.0 \%$ \\
\hline Água e luz (serviços públicos) & 9 & $5.4 \%$ \\
\hline Outros (distribuído entre 12 setores) & 59 & $35.1 \%$ \\
\hline Total & 168 & $100 \%$ \\
\hline
\end{tabular}

Tanto o convite individual feito na rede de contatos dos autores quanto o convite para participação na pesquisa feito na lista de discussão eram direcionados ao responsável pelo programa de gestão do conhecimento. Caso não existisse essa função na organização, a mensagem solicitava que o convite fosse encaminhado para o gestor de TI ou então para o gerente de RH nessa ordem. Entre os respondentes, $14,3 \%$ é composto por gerentes de TI, 13,1\% por gerente de RH, 12,5\% por administradores da intranet/portal, 8,3\% por líderes de projeto de TI, 6,0\% por líderes de projetos de GC, 6,0\% por analistas de sistemas e o restante se encontra disperso entre 14 funções relacionadas com comunicação interna e RH.

Um dos aspectos interessantes levantados pela pesquisa diz respeito ao setor responsável pela liderança dos projetos de GC. Essa questão admitia múltiplas respostas com exceção das opções "nenhum setor é diretamente responsável” e “outro setor". A Tabela 3 apresenta os resultados em ordem decrescente da freqüência (Notar que a soma será superior a 100\% por causa das respostas múltiplas). É curioso observar um empate técnico entre os setores de TI e RH provocado pelo leve predomínio da área de TI sobre a de RH no Brasil e o inverso acontecendo em Portugal. Infelizmente verifica-se ainda um percentual elevado de $20.2 \%$ para a falta de uma definição do setor responsável por GC. Os resultados também indicam que a criação de um setor específico para GC não ocorreu na maioria das organizações analisadas, sendo que a divisão de responsabilidade ocorre usualmente entre os setores de TI e RH. 
Tabela 3 - Freqüência dos setores responsáveis pela liderança de iniciativas de GC

\begin{tabular}{llll}
\hline Setor responsável pela liderança de GC & $\begin{array}{l}\text { Freqüência } \\
\text { Global }\end{array}$ & $\begin{array}{l}\text { Freqüência } \\
\text { (Brasil) }\end{array}$ & $\begin{array}{l}\text { Freqüência } \\
\text { (Portugal) }\end{array}$ \\
\hline Tecnologia da Informação & $49.1 \%$ & $53,1 \%$ & $37.3 \%$ \\
\hline Recursos Humanos & $49.1 \%$ & $57,3 \%$ & $43.3 \%$ \\
\hline Diretoria / Comitê Gerencial & $27.6 \%$ & $28.1 \%$ & $26.9 \%$ \\
\hline Comunicação & $23.3 \%$ & $25.0 \%$ & $20.9 \%$ \\
\hline $\begin{array}{l}\text { Nenhum setor específico é diretamente } \\
\text { responsável por gestão do conhecimento }\end{array}$ & $20.2 \%$ & $17.7 \%$ & $23.9 \%$ \\
\hline Biblioteca / Centro de Documentação & $16.6 \%$ & $21.9 \%$ & $9.0 \%$ \\
\hline Setor de Pesquisa e Desenvolvimento & $11.0 \%$ & $15.6 \%$ & $4.5 \%$ \\
\hline Setor Específico de Gestão do Conhecimento & $8.6 \%$ & $12.6 \%$ & $3.0 \%$ \\
\hline Outros setores & $\begin{array}{l}2.4 \%(\text { do total de } \\
\text { casos) }\end{array}$ & $\begin{array}{l}2.0 \%(\text { do total de } \\
\text { casos) }\end{array}$ & $\begin{array}{l}2.9 \% \text { (do total de } \\
\text { casos) }\end{array}$ \\
\hline
\end{tabular}

As pontuações médias obtidas para cada uma das questões sobre prática de gestão do conhecimento são apresentadas na Tabela 4 em ordem decrescente da média global. A nota 10 representava o nível máximo de atendimento à variável proposta. O número entre parênteses indica o resultado obtido para a questão de mesmo número (vide Anexo 1). Apenas para fins ilustrativos, a última coluna apresenta a diferença das médias entre Brasil e Portugal. Quando a diferença for positiva, significa que a média brasileira é melhor do que a portuguesa. Os itens em negritos destacam o país que obteve a melhor média para o quesito avaliado.

Tabela 4 - Nível de existência de práticas de GC em organizações brasileiras e portuguesas

\begin{tabular}{|c|c|c|c|c|c|c|c|}
\hline $\begin{array}{c}\text { Prática de Gestão do } \\
\text { Conhecimento }\end{array}$ & $\begin{array}{l}\text { Média } \\
\text { Global }\end{array}$ & $\begin{array}{l}\text { Desvio } \\
\text { Padrão }\end{array}$ & $\begin{array}{c}\text { Média } \\
\text { (Brasil) }\end{array}$ & $\begin{array}{c}\text { Desvio } \\
\text { Padrão } \\
\text { (Brasil) }\end{array}$ & $\begin{array}{c}\text { Média } \\
\text { (Portugal) }\end{array}$ & $\begin{array}{c}\text { Desvio } \\
\text { Padrão } \\
\text { (Portugal) } \\
\end{array}$ & $\begin{array}{c}\text { Diferença das } \\
\text { Médias (Brasil - } \\
\text { Portugal) }\end{array}$ \\
\hline Cultura colaborativa (gc4) & 5.79 & 2.98 & 5.74 & 3.21 & 5.84 & 2.66 & -0.10 \\
\hline $\begin{array}{c}\text { Socialização do } \\
\text { conhecimento }(\mathrm{gc} 6)\end{array}$ & 5.67 & 3.06 & 5.59 & 3.26 & 5.77 & 2.77 & -0.18 \\
\hline $\begin{array}{l}\text { Avaliação de atitudes de } \\
\text { compartilhamento (gc8) }\end{array}$ & 5.57 & 3.18 & 5.54 & 3.28 & 5.60 & 3.04 & -0.06 \\
\hline $\begin{array}{l}\text { Inteligência Competitiva } \\
(\mathrm{gc} 1)\end{array}$ & 5.45 & 3.13 & 5.54 & 3.37 & 5.33 & 2.78 & 0.21 \\
\hline Tomada de decisão (gc12) & 5.08 & 2.97 & 4.81 & 3.09 & 5.46 & 2.79 & -0.65 \\
\hline $\begin{array}{l}\text { Registro de lições aprendidas } \\
\text { (gc7) }\end{array}$ & 4.90 & 2.98 & 4.78 & 3.10 & 5.07 & 2.83 & -0.30 \\
\hline Orçamento de GC (gc2) & 4.81 & 3.29 & 4.77 & 3.41 & 4.87 & 3.15 & -0.11 \\
\hline $\begin{array}{l}\text { Comunidades de práticas } \\
\qquad(\mathrm{gc} 5)\end{array}$ & 4.70 & 3.17 & 4.54 & 3.46 & 4.93 & 2.74 & -0.39 \\
\hline Liderança de GC (gc9) & 4.59 & 3.36 & 4.85 & 3.66 & 4.23 & 2.89 & 0.62 \\
\hline Estratégia de GC (gc3) & 4.29 & 3.45 & 4.02 & 3.68 & 4.67 & 3.07 & -0.65 \\
\hline Projetos piloto de GC (gc10) & 4.47 & 3.28 & 4.83 & 3.49 & 3.97 & 2.91 & 0.86 \\
\hline Mensuração (gc11) & 3.98 & 3.35 & 3.93 & 3.59 & 4.06 & 3.00 & -0.13 \\
\hline
\end{tabular}


De maneira geral, as médias podem ser apenas razoáveis, sinalizando que ainda existe um longo caminho a ser trilhado para uma GC mais amadurecida. Basta constatar que nenhuma variável apresentou uma média melhor do que 6. É interessante observar que as três variáveis que apresentaram melhor desempenho para as organizações analisadas estão vinculadas com aspectos mais humanos e comportamentais da GC. A existência de políticas de avaliação de desempenho dos funcionários (gc8) que incorporam aspectos de valorização de atitudes de compartilhamento e criação do conhecimento é importante para estimular a cultura colaborativa e a socialização. No entanto, esse aspecto comportamental da GC parece ainda não ter conseguido estimular suficientemente a formação de comunidades de prática (gc5). A predominância do aspecto tácito da GC em detrimento do explícito fica mais evidente ao verificarmos o desempenho da variável associada ao registro de lições aprendidas e melhores práticas (gc7).

Já as médias das variáveis inteligência competitiva ( $\mathrm{gc} 1)$ e tomada de decisão ( $\mathrm{gc} 12)$ evidenciam que a alta competitividade dos mercados globais tem pressionado as organizações a desenvolverem instrumentos de monitoração ambiental e de construção de alianças estratégicas, ficando assim mais sintonizadas e integradas ao ambiente externo. Apesar de ainda tímidas, essas médias indicam que as organizações estão atentas à necessidade de possuir processos decisórios mais ágeis e melhor subsidiados.

Por outro lado, o fraco desempenho obtido pelas variáveis orçamento de GC (gc2), liderança de GC (gc9), estratégia de GC (gc3), projetos-piloto de GC (gc10) e mensuração (gc11) ilustra que as organizações pesquisadas, apesar de possuírem algumas boas práticas colaborativas alinhadas ao conceito da GC, ainda se encontram nos estágios iniciais da maturidade de GC. Em conjunto, essas variáveis sinalizam um alto nível de informalidade e uma carência de visibilidade estratégica para as iniciativas de GC. Os resultados apresentados indicam que as iniciativas de GC, para a maioria das organizações analisadas, não tem uma liderança clara, um orçamento definido e muito menos um plano de ação formalizado. Com isso, fica difícil implementar projetos-piloto de GC (gc10) que tenham uma maior repercussão organizacional. Assim sendo, sem liderança e sem estratégia, ainda parece muito cedo para falar de indicadores e mensuração (gc11). De uma maneira global, a maioria das iniciativas de GC das organizações analisadas poderia ser enquadrada entre os níveis inicial e repetitível do modelo KMMM.

As médias de Brasil e Portugal foram bastante parecidas, sinalizando que as iniciativas de GC encontram-se em níveis bastante similares nos dois países, com uma ligeira vantagem para Portugal. Os itens em que Portugal mais se sobressaiu em relação ao Brasil foram tomada de decisão (gc12) e estratégia de GC (gc3). Por outro lado, o Brasil obteve uma melhor média do que Portugal no aspecto da implementação de projetos de GC. Isso indica que entre as organizações brasileiras analisadas existem aquelas que implementaram projetos piloto de GC sem ter uma 
estratégia definida, sugerindo uma abordagem do tipo "aprender fazendo".

\section{Conclusões}

A conjugação desenvolvida ao longo deste artigo entre variáveis oriundas do modelo da organização do conhecimento (CHOO, 1998) e do modelo KMMM (EHMS e LANGEN, 2002) pretende contribuir para uma avaliação mais abrangente das iniciativas de GC. A pesquisa exploratória apresentada nesse artigo levantou vários aspectos que merecerão uma investigação aprofundada em trabalhos futuros. O próximo passo da pesquisa será realizar um tratamento quantitativo mais detalhado, utilizando técnicas de análise fatorial.

Entre as principais contribuições desse artigo, pode-se destacar a constatação de que a GC nas organizações analisadas encontra-se ainda nos estágios iniciais de maturidade, sendo marcada pela informalidade. Sem uma liderança clara e uma visão estratégica, as iniciativas de GC passam a depender do esforço heróico de alguns profetas. Os modelos de maturidade de GC pregam justamente o contrário, sugerindo uma padronização mínima que permita replicar as experiências piloto de GC em âmbito corporativo.

Uma surpresa positiva do trabalho foi o bom número de organizações do setor público entre os respondentes. Isso indica que vários funcionários públicos participam dos fóruns de discussão sobre GC onde o convite para participação na pesquisa foi divulgado. Portanto, o nicho "GC no setor público" continua sendo merecedor de atenção e parece caminhar em paralelo com iniciativas de governo eletrônico.

Outra constatação pertinente foi o equilíbrio de forças entre as áreas de TI e RH na liderança dos projetos em GC. Talvez seja essa a estratégia encontrada para buscar balancear os aspectos tecnológicos e humanos da GC, principalmente em situações de redução de custos quando é difícil justificar a criação de um setor específico para GC. Esse sabor de gestão de RH nas iniciativas de GC ficou evidente nessa pesquisa, pois as três práticas mais bem avaliadas de GC têm relação direta com aspectos de RH.

Um uso interessante do questionário proposto nesse artigo é aplicá-lo como um instrumento periódico, realizando uma nova avaliação da mesma organização a cada ano para verificar se houve algum avanço ou retrocesso. Após submeter a sua iniciativa de GC ao modelo de avaliação proposto, a organização terá condição de identificar ações corretivas ou evolutivas, visando atingir uma maior maturidade em suas iniciativas de GC. 


\begin{abstract}
Organizations have been deploying knowledge management initiatives whose objectives comprise organizational memory preservation, productivity increase of knowledge workers, incentive to innovation processes and better capacity to compete. On the other hand, evaluation of the results of such initiatives is a hard task due to the lack of standards related to the deployment of theoretical frameworks, and to the complex and interdisciplinary nature of knowledge management. Practitioners and scholars are working on these issues and proposing models that help organizations in developing knowledge management programs. This paper proposes a combination of the academic knowing organization model with the commercial knowledge management maturity model. The resulting model was applied in a survey with 98 Brazilian and 70 Portuguese organizations. The model variables are related to features such as collaborative culture, competitive intelligence, decision-making, knowledge creation, strategy and communities of practice. The suggested model intends to help organizations in identifying strengths and weaknesses of knowledge management programs.
\end{abstract}

Key-words: knowledge management, innovation, strategy, decision making

\title{
Referências
}

BURROWS, Glen; DRUMMOND, Damon; MARTINSONS, MARIS. Knowledge management in China. Communications of the Association of Computing Machinery, v.48, n.4, pp. 73-76, abril, 2005.

CHOO, Chun Wei. The Knowing Organization. New York: Oxford University Press, 1998.

DAVENPORT, Thomas, PRUSAK Laurence. Conhecimento empresarial: como as organizações gerenciam seu capital intelectual. Rio de Janeiro: Campus, 1998.

EHMS, Karsten; LANGEN, Manfred. Holistic development of knowledge management with Knowledge

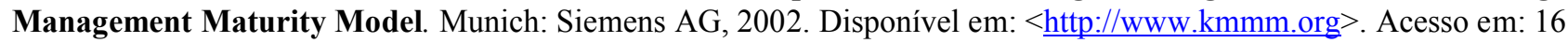
de março de 2005.

KROGH, George von, ICHIJO, Kazuo, NONAKA, Ikujiro. Facilitando a Criação do Conhecimento. Rio de Janeiro: Editora Campus, 2001.

LEONARD-BARTON, Dorothy. The wellsprings of knowledge: building and sustaining the sources of innovation. Boston: Harvard Business School Press, 1995.

NONAKA, I., TAKEUCHI, H. A Criação do Conhecimento na Empresa. São Paulo: Campus, 1997.

PAULK, Mark; WEBER, Charles; CURTIS, Bill; CHRISSIS, Mary. The Capability Maturity Model: guidelines for improving the software process. Boston: Addison-Wesley, 1995.

RODRIGUES, Hugo, ANTUNES, Adelaide M.S., DUTRA, Luís Eduardo. Análise de propostas de modelos de gestão direcionados para o conhecimento. Revista de Administração, v.38, n.1, p. 66-76, jan-mar 2003.

TEIXEIRA, Jayme. Gerenciando conhecimento. Rio de Janeiro: Editora SENAC, 2000.

TERRA. José Claúdio. Gestão do conhecimento: o grande desafio empresarial. São Paulo: Negócio Editora, 2000.

WEBER, Frithjof; WUNRAM, Michael; KEMP, Jeroen; PUDLATZ, Marc; BREDEHORST, Bernd. Standardization in Knowledge Management: Towards a common KM framework in Europe. Proceedings of Unicom Seminar, 2002. Londres. 


\section{ANEXO I}

Extrato resumido da versão brasileira do questionário utilizado na pesquisa.

$1^{\text {a }}$. Parte - Questões sobre Práticas da Gestão do Conhecimento

\begin{tabular}{|c|c|}
\hline $\begin{array}{l}\text { 1.. PARTE - Práticas da Gestão do Conhecimento } \\
\text { Responda de: }(0) \text { - Discordo totalmente Até: }(10)-\text { Concordo totalmente }\end{array}$ & $\begin{array}{l}\text { Grau de Concor- } \\
\text { dância }(0-10)\end{array}$ \\
\hline $\begin{array}{l}\text { 1. A organização dedica recursos para detectar e obter informações externas sobre } \\
\text { competidores, clientes, universidades, governo, fornecedores e associações de classe. } \\
(\mathrm{gc} 1)\end{array}$ & \\
\hline $\begin{array}{l}\text { 2. A organização possui um orçamento específico que é suficiente para assegurar o } \\
\text { desenvolvimento contínuo de suas iniciativas de gestão do conhecimento }(\mathrm{gc} 2) \text {. }\end{array}$ & \\
\hline $\begin{array}{l}\text { 3. A organização possui uma estratégia escrita de GC alinhada com os objetivos } \\
\text { empresariais. }(\operatorname{gc} 3)\end{array}$ & \\
\hline $\begin{array}{l}\text { 4. A cultura organizacional estimula a criatividade, experimentação, inovação, } \\
\text { colaboração e compartilhamento de conhecimento entre os departamentos. (gc4) }\end{array}$ & \\
\hline 5. A organização promove a criação de comunidades de práticas. (gc5) & \\
\hline $\begin{array}{l}\text { 6. A organização estimula os funcionários mais experientes a transferirem seus } \\
\text { conhecimentos para os novatos ou menos experientes. (gc6) }\end{array}$ & \\
\hline $\begin{array}{l}\text { 7. A organização mantém informações atualizadas e organizadas sobre boas práticas de } \\
\text { trabalho e lições aprendidas. (gc7) }\end{array}$ & \\
\hline $\begin{array}{l}\text { 8. As atitudes de criação e compartilhamento do conhecimento são aspectos muito } \\
\text { importantes na avaliação do desempenho dos funcionários. }(\mathrm{gc} 8)\end{array}$ & \\
\hline $\begin{array}{l}\text { 9. A organização possui um grupo multi-funcional que é responsável pelo projeto e } \\
\text { implantação de iniciativas de gestão do conhecimento. (gc9) }\end{array}$ & \\
\hline $\begin{array}{l}\text { 10. A organização já experimentou projetos piloto bem sucedidos de gestão do } \\
\text { conhecimento }(\mathrm{GC}) \text {, evidenciando a contribuição da GC para os negócios. }(\mathrm{gc} 10)\end{array}$ & \\
\hline $\begin{array}{l}\text { 11. A organização mensura os resultados de suas iniciativas de gestão do conhecimento } \\
\text { através de indicadores qualitativos e quantitativos. (gc11) }\end{array}$ & \\
\hline $\begin{array}{l}\text { 12. Informações sobre boas práticas de trabalho, erros e/ou defeitos, documentação de } \\
\text { projetos e lições aprendidas são levadas em consideração no momento da tomada de } \\
\text { decisões. }(\mathrm{gc} 12)\end{array}$ & \\
\hline
\end{tabular}

\section{$2^{\text {a }}$. Parte - Dados Sócio-Econômicos}

Instruções: Nessa parte final do questionário, as questões dizem respeito ao porte e ramo de atividade de sua organização, bem como alguns dados profissionais.

1 - Indique o principal ramo de atividade de sua organização. (Por favor, selecione uma única opção.)
( ) Agricultura / pecuária
( ) Indústria farmacêutica / cosméticos
( ) Água, energia ou luz (serviços
( ) Indústria química / petróleo públicos)

( ) Alimentos e bebidas

( ) Comércio atacadista

( ) Metalurgia / siderurgia /mineração

( ) Comércio varejista

( ) Mídia e comunicação

( ) Construção civil

( ) Saúde

( ) Consultoria

( ) Seguro

( ) Educação

( ) Setor financeiro (bancos, corretoras)

( ) Governo

( ) Tecnologia da Informação

( ) Indústria automotiva

( ) Telecomunicações

( ) Indústria eletroeletrônica

( ) Transporte ou logística

( ) Outro ramo:

2 - Quantos funcionários sua organização possui ?

( ) $0-100$

( ) 101-500

( ) 501-1.000

( ) $1.001-5.000$

( ) 5.001-10.000

( ) $10.001-20.000$

( ) Mais de 20.000 
3 - Quais são os grupos/departamentos responsáveis pelos projetos de gestão do conhecimento em sua organização ? (Essa questão permite a marcação de mais de uma opção. Por favor, marque as opções que refletirem a realidade da sua organização.)

( ) Biblioteca / Centro de Documentação

( ) Comunicação

( ) Diretoria / Comitê Gerencial

( ) Recursos Humanos

( ) Setor de Pesquisa e Desenvolvimento

( ) Setor Específico de Gestão do Conhecimento

( ) Tecnologia da Informação

( ) Nenhum setor específico é diretamente responsável por gestão do conhecimento

( ) Outro setor, por favor especique

4 - Qual é a opção que melhor descreve sua função atual na organização ? (Por favor, selecione uma única opção.)

( ) Administrador da Intranet / Webmaster

( ) Analista de Recursos Humanos

( ) Analista de Sistemas

( ) Analista de Suporte

( ) Auxiliar Administrativo / Suporte Administrativo

( ) Gerente de Gestão do Conhecimento (CKO)

( ) Gerente de Recursos Humanos

( ) Gerente de Tecnologia da Informação (CIO)

( ) Líder de Projetos de Gestão do Conhecimento

( ) Líder de Projetos de Tecnologia da Informação

( ) Outra função, por favor especifique:

\section{Dados completos de todos os autores:}

\section{Nome completo: Rodrigo Baroni de Carvalho}

Filiação institucional: Universidade FUMEC

Departamento: FACE-FUMEC

Função ou cargo ocupado: Professor Adjunto

Endereço completo para correspondência: Rua Ramalhete 313 - apto. 201 - Bairro Anchieta CEP: 30.310-310 - Belo Horizonte - MG

Telefones para contato: (0xx31) 3287-2563 / (0xx31) 9122-6948

e-mail: baroni@fumec.com.br; rodbaroni@yahoo.com.br

\section{Nome completo: Marta Araújo Tavares Ferreira}

Filiação institucional: Universidade Federal de Minas Gerais / Centro Universitário U.N.A

Departamento: Escola de Ciência da Informação (UFMG) / Mestrado em Turismo (U.N.A)

Função ou cargo ocupado: Professora

Endereço completo para correspondência: Rua Chicago 337 - apto. 71 - Bairro Sion - CEP: 30.315-520 - Belo Horizonte - MG

Telefones para contato: (0xx31) 3285-1674

e-mail:maraujo@eci.ufmg.br 


\section{Nome completo: Ricardo Vidigal da Silva}

Filiação institucional: Universidade de Évora

Departamento: Escola de Administração

Função ou cargo ocupado: Professor convidado

Endereço completo para correspondência: Rua Almada Negreiros 14, Vila Lusitano, código postal: 7005-288 - Évora - Portugal

e-mail: Ricardo.silva@mail.telepac.pt

Recebido para publicação em: 19/06/06

Aceito para publicação em: 04/09/06 